\title{
Short neutrino burst from failed supernovae as a probe of dense matter with hyperon mixture
}

\author{
Kohsuke Sumiyoshi* ${ }^{\dagger}$ \\ Numazu College of Technology, Ooka 3600, Numazu, Shizuoka 410-8501, Japan \\ E-mail: sumienumazu-ct.ac.jp
}

\section{Chikako Ishizuka}

Department of Cosmosciences, Graduate School of Science,

Hokkaido University, Sapporo 060-0810, Japan

\section{Akira Ohnishi}

Yukawa Institute for Theoretical Physics, Kyoto University, Kyoto 606-8502, Japan

\section{Shoichi Yamada}

Science and Engineering \& Advanced Research Institute for Science and Engineering,

Waseda University, Okubo, 3-4-1, Shinjuku, Tokyo 169-8555, Japan

\section{Hideyuki Suzuki}

Faculty of Science and Technology, Tokyo University of Science,

Yamazaki 2641, Noda, Chiba 278-8510, Japan

\begin{abstract}
We study the gravitational collapse of non-rotating massive stars $\left(40-50 \mathrm{M}_{\odot}\right)$ and the associated neutrino burst by general relativistic $v$-radiation hydrodynamics. We show that the neutrino burst from the accreting proto-neutron star till the black hole formation is short and energetic in a situation of failed explosion. This unique characteristics is a hallmark of black hole formation and distinguishable from the neutrino signal of ordinary supernovae. Adopting the sets of physical equation of state of dense matter, we demonstrate that the duration of neutrino emission depends crucially on the equation of state. Future detection of neutrinos from the failed supernovae can be used to probe the stiffness of dense matter with possible emergence of hyperons.
\end{abstract}

10th Symposium on Nuclei in the Cosmos

July 27 - August 12008

Mackinac Island, Michigan, USA

\footnotetext{
*Speaker.

$\dagger$ This work is supported partially by the Grants-in-Aid for the Scientific Research (18540291, 18540295, 19104006, 19540252) of the MEXT of Japan. The numerical simulations were performed at CfCA in NAOJ, JAEA, YITP (Kyoto U.) and RCNP (Osaka U.).
} 


\section{Black hole formation in failed supernovae}

The fate of massive stars $\left(\geqslant 20 \mathrm{M}_{\odot}\right)$ attracts recent attention to understand the diversity of core-collapse supernovae. Whereas the massive stars of $10-20 \mathrm{M}_{\odot}$ usually lead to the ordinary supernova explosion with neutron star formation, more massive stars lead to the black hole formation associated with hypernovae or faint supernovae [1]. We focus here on the fate of non-rotating massive stars in the range of $40-50 \mathrm{M}_{\odot}$ without explosion as a limiting case of faint supernovae (ex. SN 1997D and SN 1999br). These failed supernovae do not show any optical display but are associated with the characteristic emission of neutrinos. The failed supernovae can be a substantial fraction of core-collapse supernovae and may be observed in the survey of disappearance of massive stars [2].

Since the iron core is too large for the massive stars of $\sim 40 \mathrm{M}_{\odot}$, the explosion is not possible intrinsically under the spherically symmetric condition. Starting from the iron core collapse, a proto-neutron star is born after the bounce. The material from outer layers accretes intensively onto the proto-neutron star, which becomes massive and compact. The mass of proto-neutron star increases continuously beyond the typical neutron star mass. When it reaches the critical mass, that is determined by EOS, the dynamical collapse occurs to form a black hole. Accordingly, the neutrino burst emitted from the proto-neutron star is terminated by the black hole formation. The duration of neutrino burst is found to be $\sim 1 \mathrm{~s}$ from the core bounce [3]. We note that this scenario is different from the one in ordinary supernovae, in which quasi-static proto-neutron stars cool down gradually by emitting neutrinos for $\sim 20 \mathrm{~s}$ after the successful launch of shock wave.

We demonstrate that the neutrino bursts till the black hole formation in failed supernovae can be used to probe the properties of dense matter with a possible mixture of hyperons. We show that the neutrino emission from non-rotating failed supernovae is in the range of 0.4-1.5 s with the rapid increase of average energies during the dynamical collapse [4, 5]. These features are clearly different from those of ordinary supernovae and enable us to identify the black hole formation. The duration of neutrino burst depends crucially on the EOS of dense matter and is less dependent on the progenitors. Therefore, a terrestrial detection of the short neutrino burst may constrain the stiffness of nuclear matter and the appearance of new degrees of freedom in dense matter [6].

\section{Numerical simulations of general relativistic $v$-radiation hydrodynamics}

In order to explore the neutrino burst from failed supernovae and its relation to the equation of state, we have performed systematically a series of numerical simulations by solving the general relativistic $v$-radiation hydrodynamics. We follow the dynamics starting from the initial collapse of iron core, the core bounce, the propagation of shock wave, and the evolution of accreting proto-neutron star till the black hole formation. We adopt the progenitor models obtained by the evolutionary calculation of massive stars by three groups [7, 8, 9] as initial models. The same set of weak interaction rates as in [10] is adopted for the current simulations. Details of our numerical simulations can be found in [4, 10].

We adopt the three sets of supernova EOS to examine the influence of EOS on the neutrino signal. The EOS by Shen et al. [11, 12] (Shen-EOS) is derived by the relativistic mean field framework with the data of unstable nuclei. It provides a rather stiff EOS having the maximum mass of cold neutron stars to be $2.2 \mathrm{M}_{\odot}$. The EOS by Lattimer and Swesty [13] (LS-EOS) is 

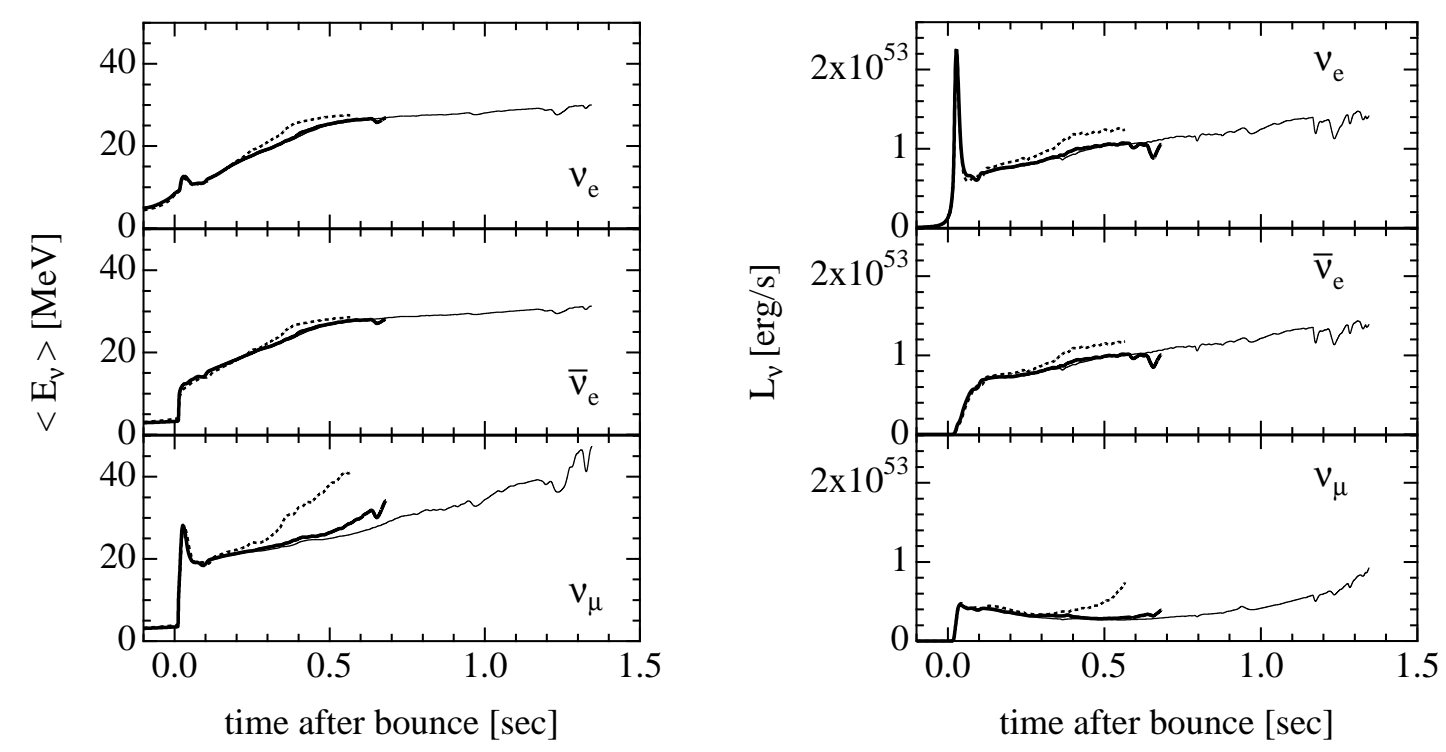

Figure 1: Time profiles of average energies and luminosities of neutrinos are shown for models W40S (thin solid), W40L (dotted) and W40I (thick solid).

obtained by the extension of the compressible liquid model with a density dependence of energy based on the Skyrme-interaction. We have chosen the case of incompressibility of $180 \mathrm{MeV}$ among the three choices in the LS-EOS as a representative of soft EOS. The maximum mass of cold neutron stars is $1.8 \mathrm{M}_{\odot}$ in this case.

In addition, we adopt a new set of supernova EOS including hyperons. The hyperonic $\operatorname{EOS}^{1}$ was recently developed by Ishizuka et al. [14] (Ishizuka-EOS), extending the Shen-EOS to SU(3) symmetry to include the octet baryons. It should be stressed that the Ishizuka-EOS is connected smoothly with the Shen-EOS by adopting the same interaction for the nucleon sector. The interactions of hyperons are determined by the recent experimental data of hypernuclei. As for the $\Sigma^{-}$-potential in nuclear matter, we choose the repulsive value $(+30 \mathrm{MeV})$, which is recommended lately. The maximum mass of cold neutron stars is $1.6 \mathrm{M}_{\odot}$, which is smaller than that of the ShenEOS. Because of the mixture of hyperons, the EOS becomes soft at high densities and the maximum mass is reduced.

\section{Characteristics of neutrino bursts from non-rotating failed supernovae}

We explore the influence of the equation of state on the collapse of central core of a $40 \mathrm{M}_{\odot}$ star by Woosley and Weaver [7] at first. We put model names as W40S, W40L and W40I for the cases with three sets of EOS: the Shen-EOS, LS-EOS and Ishizuka-EOS, respectively. We found that the massive proto-neutron star is formed right after the core bounce under the intense accretion. Its mass increases rapidly and exceeds $\sim 2 \mathrm{M}_{\odot}$ within $\sim 0.5 \mathrm{~s}$ after the bounce. The re-collapse occurs earlier in W40L and W40I than that in W40S when the proto-neutron star mass reaches the critical

\footnotetext{
${ }^{1}$ The data tables with some options on hyperon interactions and inclusion of pions are available for public use.
} 


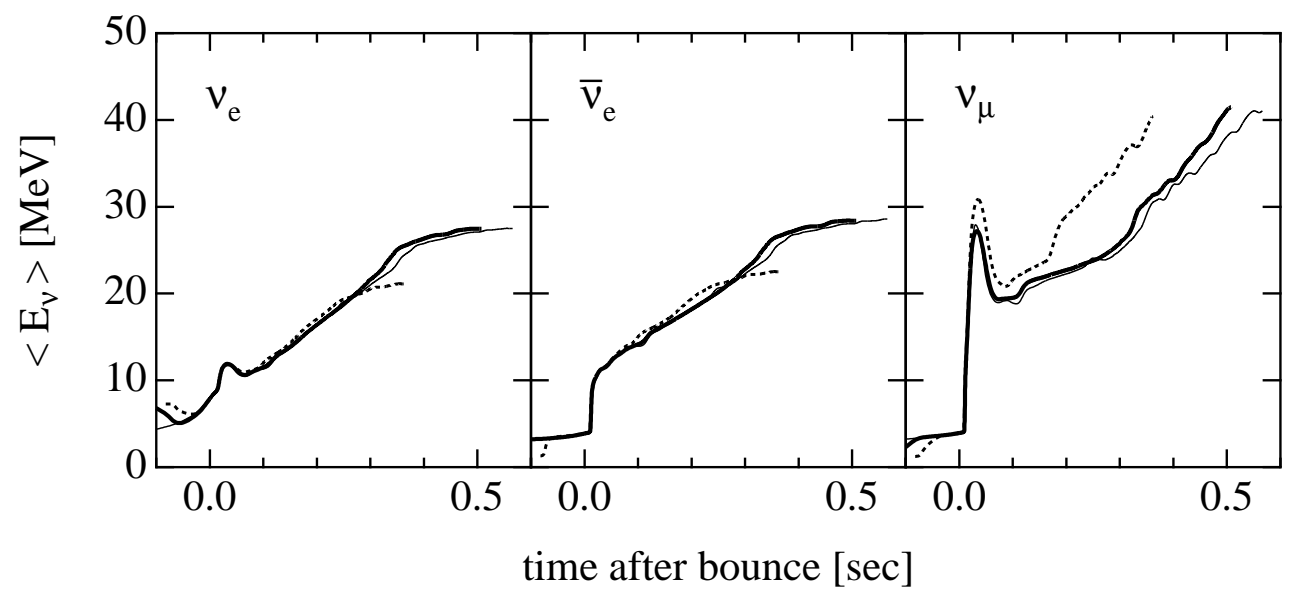

Figure 2: Time profiles of average energies of neutrinos are shown for models W40L (thin solid), T50L (thick solid) and H40L (dotted).

mass determined by the adopted EOS. The critical mass is largest for the Shen-EOS and, therefore, the duration of accreting proto-neutron star is longest among the three cases.

In Fig. 1, we show the time profiles of emitted neutrinos $\left(v_{e}, \bar{v}_{e}\right.$ and $\left.v_{\mu}\right)$ during the evolution up to the black hole formation. The duration of neutrino bursts reflects clearly the timing of the black hole formation. This difference of duration can be used to probe the stiffness of EOS. It is remarkable to see that the average energies (left panels) increase rapidly toward the end. Since $v_{e}$ and $\bar{v}_{e}$ are dominantly produced by the accretion around the surface of proto-neutron star, their average energies are similar among the three EOS sets. On the other hand, the average energy of $v_{\mu}$ reflects the difference of temperature in more deeper regions of the contracting proto-neutron star, depending on the EOS. The luminosities (right panels) show also increasing behaviors due to the increase of accretion luminosity and thermal energies.

Comparing the models with and without hyperons (W40S and W40I) in the same nuclear framework, we found that the energies and luminosities are almost the same, but only the duration is different. This is due to the sudden appearance of hyperons, which induce the dynamical recollapse [5]. Therefore, if one can fix the framework for the nuclear matter, one may be able to extract when the hyperon mixture occurs in dense matter from the duration of neutrino burst.

In order to establish the neutrino burst as a probe of EOS, we investigate the dependence on progenitor models. We have performed additionally two simulations with the LS-EOS adopting a $40 \mathrm{M}_{\odot}$ star by Hashimoto [8] (H40L) and a $50 \mathrm{M}_{\odot}$ star by Tominaga et al. [9] (T50L). In Fig. 2 , we show the time profiles of average energies of neutrinos for the three models. The duration of neutrino burst is well within the range of 0.4-0.6 s for the LS-EOS even if we change the progenitor. Among the three models, the accretion rate is most high in $\mathrm{H} 40 \mathrm{~L}$ and, therefore, the duration is most short. The average energy of $v_{\mu}$ in H40L increases faster than the others according to rapid increase of temperature. The dependence on the progenitor is rather weak as compared to the large uncertainty from the EOS. This is because the massive stars of $\sim 40 \mathrm{M}_{\odot}$ have the density profiles which lead to similar history of accretion rates. These findings strengthen the argument to 
use the neutrino signal to constrain the EOS, however, one should study further the wide variety of massive stars since our study is limited for three selected models.

\section{Summary}

We demonstrated the neutrino burst in failed supernovae from massive stars of $\sim 40 \mathrm{M}_{\odot}$ has attractive features for astrophysics and nuclear physics. The neutrino emission is short with increasing energy and, therefore, distinguishable from neutrinos from ordinary supernovae. The duration of neutrino burst depends crucially on the EOS of dense matter. The future detection of neutrinos in failed supernovae will enable us to constrain the stiffness of EOS. Because the density and temperature becomes very high during the contracting proto-neutron stars toward the black hole, the new degrees of freedom such as hyperons and quarks may appear [15]. We have revealed that the hyperons appear just before the black hole formation. Its neutrino signal is similar to the counterpart with nucleonic EOS, but the duration becomes solely short. It may be able to constrain the threshold density for the mixture of hyperons from neutrino detections. In contrast, the dependence on the progenitors is found rather weak as compared with the EOS dependence. With the information on progenitor in the planned survey of disappearance of massive stars, we envisage the future observation of the short neutrino burst from the quiet death of massive stars may provide the information of properties of dense matter. Evaluation of event numbers of neutrinos, taking into account neutrino oscillations, at the terrestrial detectors will be published elsewhere [16].

\section{References}

[1] K. Nomoto et al., in "Supernova 1987A: 20 Years After: Supernovae and Gamma-Ray Bursters" eds. S. Immler, K. Weiler and R. McCray (AIP, 2007); arXiv:0707.2187.

[2] C. S. Kochanek et al., Astrophys. J., (2008) in press; arXiv:0802.0456.

[3] K. Sumiyoshi, S. Yamada, H. Suzuki and S. Chiba, Phys. Rev. Lett. 97 (2006) 091101.

[4] K. Sumiyoshi, S. Yamada and H. Suzuki, Astrophys. J. 667 (2007) 382.

[5] K. Sumiyoshi, S. Yamada and H. Suzuki, Astrophys. J. 688 (2008) in press.

[6] K. Sumiyoshi, C. Ishizuka, A. Ohnishi, S. Yamada and H. Suzuki, Astrophys. J. Lett. (2008) submitted.

[7] S. E. Woosley and T. Weaver, Astrophys. J. Suppl. 101 (1995) 181.

[8] M. Hashimoto, Prog. Theor. Phys. 94 (1995) 663.

[9] N. Tominaga, H. Umeda and K. Nomoto, Astrophys. J. Suppl. 660 (2007) 516.

[10] K. Sumiyoshi, S. Yamada, H. Suzuki, H. Shen, S. Chiba and H. Toki, Astrophys. J. 629 (2005) 922.

[11] H. Shen, H. Toki, K. Oyamatsu and K. Sumiyoshi, Nucl. Phys. A637 (1998) 435.

[12] H. Shen, H. Toki, K. Oyamatsu and K. Sumiyoshi, Prog. Thoer. Phys. 100 (1998) 1013.

[13] J. M. Lattimer and F. D. Swesty, Nucl. Phys. A535 (1991) 331.

[14] C. Ishizuka, A. Ohnishi, K. Tsubakihara, K. Sumiyoshi and S. Yamada, J. Phys. G 35 (2008) 085201.

[15] K. Nakazato, K. Sumiyoshi and S. Yamada, Phys. Rev. D77 (2008) 103006.

[16] K. Nakazato, K. Sumiyoshi, H. Suzuki and S. Yamada, in preparation. 